\title{
Leveraging Label-Specific Discriminant Mapping Features for Multi-Label Learning
}

\author{
YUMENG GUO, Tongji University and The Hong Kong Polytechnic University \\ FULAI CHUNG, The Hong Kong Polytechnic University \\ GUOZHENG LI, China Academy of Chinese Medical Sciences and Tongji University \\ JIANCONG WANG and JAMES C. GEE, University of Pennsylvania
}

\begin{abstract}
As an important machine learning task, multi-label learning deals with the problem where each sample instance (feature vector) is associated with multiple labels simultaneously. Most existing approaches focus on manipulating the label space, such as exploiting correlations between labels and reducing label space dimension, with identical feature space in the process of classification. One potential drawback of this traditional strategy is that each label might have its own specific characteristics and using identical features for all label cannot lead to optimized performance. In this article, we propose an effective algorithm named LSDM, i.e., leveraging label-specific discriminant mapping features for multi-label learning, to overcome the drawback. LSDM sets diverse ratio parameter values to conduct cluster analysis on the positive and negative instances of identical label. It reconstructs label-specific feature space which includes distance information and spatial topology information. Our experimental results show that combining these two parts of information in the new feature representation can better exploit the clustering results in the learning process. Due to the problem of diverse combinations for identical label, we employ simplified linear discriminant analysis to efficiently excavate optimal one for each label and perform classification by querying the corresponding results. Comparison with the state-of-the-art algorithms on a total of 20 benchmark datasets clearly manifests the competitiveness of LSDM.
\end{abstract}

CCS Concepts: • Computing methodologies $\rightarrow$ Learning latent representations;

Additional Key Words and Phrases: Machine learning, multi-label learning, label specific features

\footnotetext{
This work was partially supported by the Natural Science Foundation of China under grant no. 61871141, the GRF, UGC under project Hong Kong PolyU 152039/14E, and the Central Research Grant, Hong Kong PolyU under project G-YBVT, the International Exchange Program for Graduate Students, Tongji University (No. 2017010007), National Key R\&D Program of China (No. 2017YFC1703501), the Fundamental Research Funds for the Central public welfare research institutes (No. ZZ0908032).

Authors' addresses: Y. Guo, Department of Control Science and Engineering, Tongji University, Shanghai, China; Department of Computing, The Hong Kong Polytechnic University, Kowloon, Hong Kong; email: yumeng_guo@foxmail.com; F. Chung, Department of Computing, The Hong Kong Polytechnic University, Kowloon, Hong Kong; email: cskchung@comp.polyu.edu.hk; G. Li, Data Center of Traditional Chinese Medicine, China Academy of Chinese Medical Sciences, Beijing, China; Department of Control Science and Engineering, Tongji University, Shanghai, China; email: drgzli@gmail.com; J. Wang and J. C. Gee, Penn Image Computing and Science Laboratory, Department of Radiology, University of Pennsylvania, Philadelphia, USA; emails: jiancong.wang@pennmedicine.upenn.edu, gee@upenn.edu.

Permission to make digital or hard copies of all or part of this work for personal or classroom use is granted without fee provided that copies are not made or distributed for profit or commercial advantage and that copies bear this notice and the full citation on the first page. Copyrights for components of this work owned by others than the author(s) must be honored. Abstracting with credit is permitted. To copy otherwise, or republish, to post on servers or to redistribute to lists, requires prior specific permission and/or a fee. Request permissions from permissions@acm.org.

(C) 2019 Copyright held by the owner/author(s). Publication rights licensed to ACM.

1556-4681/2019/04-ART24 \$15.00

https://doi.org/10.1145/3319911
} 


\section{ACM Reference format:}

Yumeng Guo, Fulai Chung, Guozheng Li, Jiancong Wang, and James C. Gee. 2019. Leveraging Label-Specific Discriminant Mapping Features for Multi-Label Learning. ACM Trans. Knowl. Discov. Data 13, 2, Article 24 (April 2019), 23 pages.

https://doi.org/10.1145/3319911

\section{INTRODUCTION}

Traditional single-label learning deals with the problem where each instance is associated with only one class label. However, in various real-world applications, multi-label examples exist as shown in Figure 1, where each instance is associated with multiple class labels simultaneously [35], and appear also in areas like text categorization where each document may belong to several topics [31], [30], [7], bioinformatics where each gene may be associated with a set of functional classes [1], [5], [37], and scene recognition where each image may demonstrate several semantic classes [3], [4], [36]. Multi-label learning aims to build classification models for multi-label objects.

For the multi-label learning problem, let $\mathcal{X}=\mathbb{R}^{d}$ denote the $d$-dimensional feature space and $\mathcal{Y}=\mathbb{R}^{q}\left(\left\{l_{k} \in\{0,1\} \mid 1 \leq k \leq q\right\}\right)$ denote the $q$-dimensional label space. Each instance $\boldsymbol{x} \in \mathcal{X}$ matches a subset of labels $\boldsymbol{y} \subseteq \mathcal{Y}$, which can be equivalently written in the form of a binary vector $\boldsymbol{y} \in\{0,1\}^{q}$, with each bit $l_{k}$ indicating the relevant or irrelevant label. Then, the goal of multilabel learning is to build a classier $h: \mathcal{X} \rightarrow 2^{\mathcal{Y}}$ which maps an instance to a subset of labels. One common strategy adopted by existing approaches is manipulating label space $\mathcal{Y}$, such as exploiting correlations between labels and reducing label space dimension, with identical feature representation of the instance, i.e., $\boldsymbol{x}$, to finish the classification task. Although many algorithms [45], [22], [15] have been designed for this strategy, it might only explore partial essence of multi-label learning. Videlicet, it might be suboptimal as the specific characteristics of each label cannot be distinguished from each other. For example, in automatic image annotation, suppose grassland and elephant are two possible classes appearing in the label space, intuitively, color-based features would be preferred in discriminating grassland and non-grassland images, while texture-based features would be preferred in discriminating elephant and non-elephant images.

Encouragingly, a multi-label learning algorithm named LIFT [41], [42] has been recently proposed to explore the label-specific features [16], i.e., the most pertinent and discriminative features for each class label. For each class label $l_{k} \in \mathcal{Y}$, LIFT performs cluster analysis on the positive and negative training instances, and then reconstructs training and testing feature spaces specific to each label $l_{k}$ by querying the clustering results. The label-specific features of LIFT are represented by distances between the original instances and the cluster centers. However, it still has two drawbacks. First, it utilizes identical ratio parameter to control the number of clusters for each label, i.e., different cluster centers corresponding to different reconstructed feature spaces, which ignores the differences between labels. Second, it only uses the distance information which does not exploit the clustering results comprehensively. Inspired by this work, we ulteriorly explore the reconstructed feature space based on label-specific information to overcome the drawbacks of LIFT.

In this article, we propose to leverage label-specific discriminant mapping features for multi-label learning and derive a corresponding algorithm called LSDM. It sets more different values of ratio parameter to generate several groups of cluster centers for each label. It also reconstructs several feature spaces specific to each label by conducting cluster analysis on its positive and negative instances. The label-specific features of LSDM includes distance information as in LIFT and new additions represented by the weights of using the cluster centers to linearly represent the original instances. The distance mapping features mainly contain far and near information between 


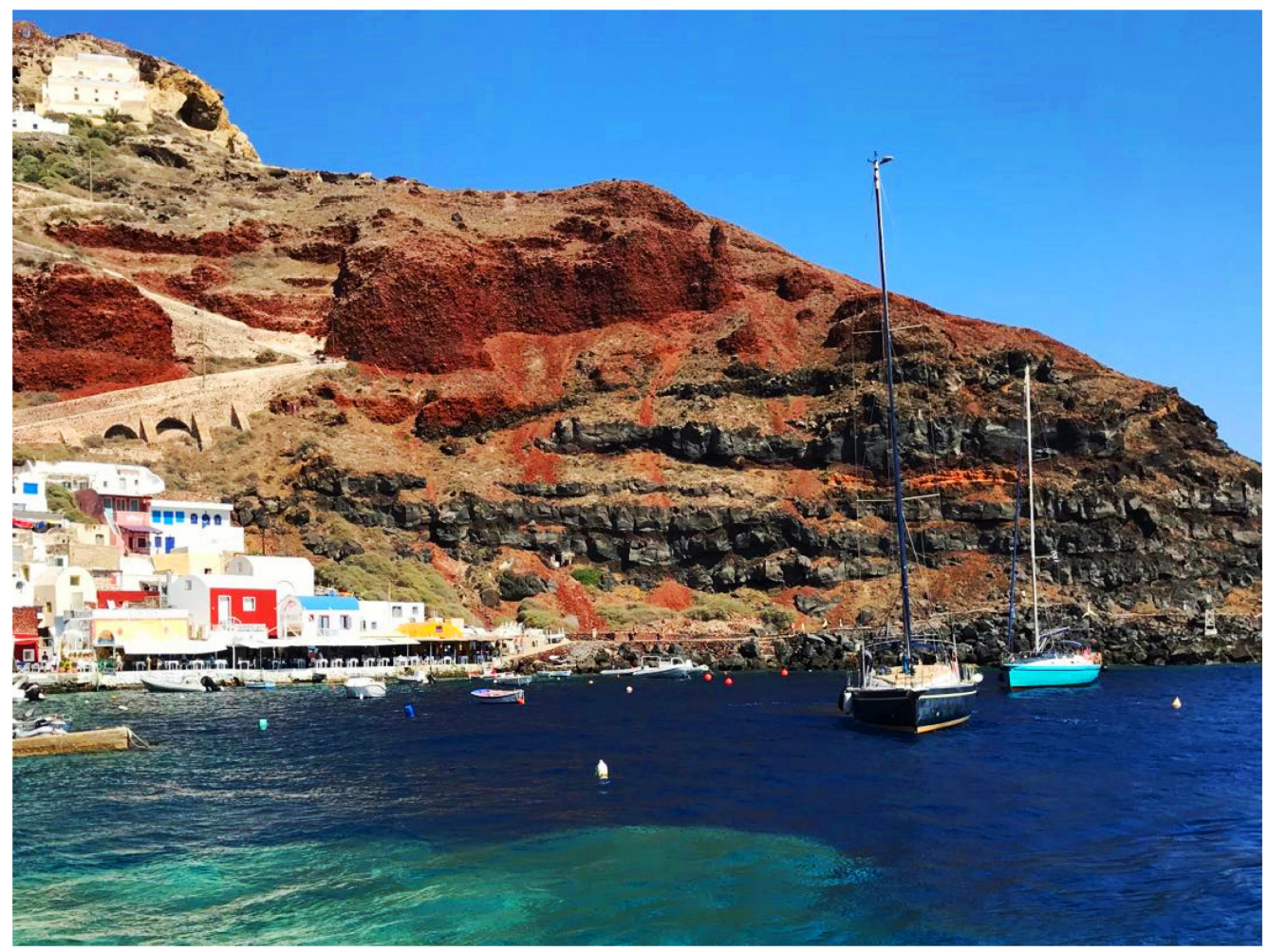

Fig. 1. An exemplar natural scene image which has been annotated with multiple labels: sky, mountain, house, boat, water (authors' own photograph).

instances and the cluster centers, while linear representation features can describe the spatial topological information between them. Through empirical test, we find the combination of the two types of features can better exploit the clustering results.

For the ratio parameter setting, i.e., using several values for each label simultaneously, we attempt to excavate the optimally reconstructed feature space corresponding to ratio value in an efficient manner. An intuitive method to handle this problem is employing classifiers. While the number of ratio value and size of dataset are large, using classifiers should bring high computational complexity leading to time-consuming process. Here, we make an assumption that if a reconstructed feature space for label $l_{k}$ is good, the distances within positive or negative instances are small and between positive and negative instances are large. Due to this assumption, we utilize the method of simplified linear discriminant analysis (sLDA), which only computes two mean values and scatters of the positive and negative training instances based on the reconstructed feature space for each label without mapping to one-dimension space (dimension reduction), in order to deal with it efficiently as a compromise.

Briefly, LSDM learns from multi-label data with five intuitive and simplified steps. First, for each class label $l_{k} \in \mathcal{Y}$, cluster analysis is performed on its positive and negative training instances; Second and third, several reconstructed feature spaces based on distance mapping and linear representation with respect to $l_{k}$ are generated by querying different clustering results. Fourth, sLDA is employed to excavate optimal one from different reconstructed feature spaces of identical class label efficiently. At last, a family of $q$ classifiers are learned from the excavated results. 
The rest of this article is organized as follows. Section 2 reviews some existing multi-label learning approaches. Section 3 presents the proposed LSDM algorithm. Section 4 presents the design of the experiment. Section 5 reports comparative experimental results over a wide range of multilabel datasets. Finally, Section 6 concludes and discusses several issues for future work.

\section{RELATED WORK}

Recently, multi-label learning has received rapidly increasing attention from machine learning and pattern recognition communities, due to its widely existing applications in real world. There is a rich body of work on the research of multi-label learning. Generally, according to the popular taxonomy presented in [33], the existing approaches can be categorized into two classes, namely, problem transformation approaches and algorithm adaptation approaches. On the other hand, according to the order of label correlations, the could be roughly categorized into three major classes [43], namely, first-order, second-order, and high-order approaches.

Problem transformation approaches tackle multi-label learning problems by turning them into one or more single-label learning problems that are solved with a single-label learning algorithm. Thus, many conventional single-label algorithms can be employed in this class, such as support vector machines, $k$-nearest neighbor and decision trees [9], and so on. For first order, the binary relevance (BR) approach [2], which decomposes a multi-label learning problem into $q$ independent binary classification problems, is simple and effective, but it ignores label correlation. For secondorder or high order, the label powerset (LP) approach [33] considers each unique set of labels as a new label, then treats them as a multi-class problem. Compared with BR, LP can utilize the label correlations in the training data. It is possible to generate a larger number of new labels with limited training examples for them. Also, it cannot predict unseen label sets. Some approaches have been proposed to overcome these problems, such as random $k$ labelsets [34] and multi-label classification using ensembles of pruned sets [27]. There is also an approach which is called calibrated label ranking (CLR) [12]. CLR introduces an artificial calibration label that, in each example, to separate the relevant from the irrelevant labels. The major merit of problem transformation approaches lies in their operational flexibility which combines existing single-label algorithms and is conceptually simple to boost the algorithm design, while the effectiveness of these approaches might be suboptimal.

Algorithm adaptation approaches tackle multi-label learning directly by adapting single-label algorithms to multi-label cases. The process of training classifiers and predicting an unseen instance in this kind of algorithms is similar to traditional single-label algorithms. The major merit of algorithm adaptation approaches is that they can utilize the characteristics of a multi-label learning problem in a more concise and elegant way. Especially, these approaches exploit secondorder (pairwise) or high-order label correlations. For second-order approaches, they can utilize the ranking criterion, such as the multi-label pairwise perceptron [23], or the co-occurrence patterns, such as the two-stage voting architecture [24]. For high-order approaches, they can impose all other class labels' influences on each label or part of class labels (label subsets), e.g., utilizing hypothesis of linear combination: instance-based learning and logistic regression (IBLR-ML and IBLR-ML+) [8], nonlinear mapping: dependent binary relevance [25], shared subspace [20], randomly selecting the label subsets [21], and utilizing graph structure to determine the specific label subsets: conditional dependency networks [13]. Obviously, algorithm adaptation approaches could take the advantage of strong label correlations to a certain extent, while suffering high computational complexities.

A common property of existing approaches is that they handle multi-label learning problem mainly by focusing on the perspective of output space. However, new type of approaches have been proposed which exploit label-specific features are exploited to benefit the discrimination of 
Table 1. Summary of Major Mathematical Notations

\begin{tabular}{|c|c|}
\hline Notations & Mathematical meanings \\
\hline$x$ & $d$-dimensional feature space $\mathbb{R}^{d}$ \\
\hline$y$ & $q$-dimensional label space $\mathbb{R}^{q}\left(\left\{l_{k} \in\{0,1\} \mid 1 \leq k \leq q\right\}\right)$ \\
\hline$x$ & $d$-dimensional feature vector $\left(x_{1}, x_{2}, \ldots, x_{d}\right)^{\mathrm{T}} \quad(x \in \mathcal{X})$ \\
\hline$y$ & Label set associated with $\boldsymbol{x}(\boldsymbol{y} \subseteq \boldsymbol{y})$ \\
\hline$\widetilde{D}$ & Multi-label training set $\left\{\left(\boldsymbol{x}_{i}, \boldsymbol{y}_{i}\right) \mid 1 \leq i \leq m\right\}$ \\
\hline $\mathcal{P}_{k}\left(\mathcal{N}_{k}\right)$ & $\begin{array}{l}\text { Training set's positive (negative) instances set according to label } l_{k} \\
\left\{\boldsymbol{x}_{i} \mid\left(\boldsymbol{x}_{i}, \boldsymbol{y}_{i}\right) \in \mathcal{D}, l_{k} \in \boldsymbol{y}_{i}\right\}\left(\left\{\boldsymbol{x}_{i} \mid\left(\boldsymbol{x}_{i}, \boldsymbol{y}_{i}\right) \in \mathcal{D}, l_{k} \notin \boldsymbol{y}_{i}\right\}\right)\end{array}$ \\
\hline $\boldsymbol{p}_{k}\left(\boldsymbol{n}_{k}\right)$ & Partition center for $\mathcal{P}_{k}\left(\mathcal{N}_{k}\right)$ \\
\hline$|\cdot|$ & $|\mathcal{A}|$ returns the cardinality of set $\mathcal{A}$ \\
\hline$\lceil\cdot\rceil$ & $\lceil a\rceil$ returns the retained integer of number $a$ \\
\hline $\mathfrak{L}$ & Binary learner for classifier $\mathrm{c}$ induction \\
\hline$\phi_{k}(\cdot)$ & $\begin{array}{l}\phi_{k}(\boldsymbol{x}) \text { returns the reconstructed label-specific features for label } l_{k} \text { and instance } \boldsymbol{x} \\
\text { which is the concatenation of distance mapping features } \phi_{k}^{\prime} \text { and linear } \\
\text { representation features } \phi_{k}^{\prime \prime}\end{array}$ \\
\hline$\beta$ & A ratio parameter controlling the number of retained clusters $\beta \in[0,1]$ \\
\hline$\phi_{k}^{\beta}(\cdot)$ & A proper feature mapping according to label $l_{k}$ and one $\beta$ value \\
\hline $\mathcal{P}_{k}^{\beta}\left(\mathcal{N}_{k}^{\beta}\right)$ & $\begin{array}{l}\text { The reconstructed training set's positive (negative) instances set according to label } \\
l_{k} \text {, one } \beta \text { value and } \phi_{k}^{\beta}(\cdot)\left\{\phi_{k}^{\beta}\left(\boldsymbol{x}_{i}\right) \mid\left(\phi_{k}^{\beta}\left(\boldsymbol{x}_{i}\right), \boldsymbol{y}_{i}\right) \in \mathcal{D}, l_{k} \in \boldsymbol{y}_{i}\right\}\left(\left\{\phi_{k}^{\beta}\left(\boldsymbol{x}_{i}\right) \mid\left(\phi_{k}^{\beta}\left(\boldsymbol{x}_{i}\right), \boldsymbol{y}_{i}\right) \in\right.\right. \\
\left.\left.\mathcal{D}, l_{k} \notin \boldsymbol{y}_{i}\right\}\right)\end{array}$ \\
\hline $\boldsymbol{m}_{p k}^{\beta}\left(\boldsymbol{m}_{n k}^{\beta}\right)$ & The feature vectors' mean value of $\mathcal{P}_{k}^{\beta}\left(\mathcal{N}_{k}^{\beta}\right)$ \\
\hline$s_{p k}^{\beta}\left(s_{n k}^{\beta}\right)$ & The scatters of $\mathcal{P}_{k}^{\beta}\left(\mathcal{N}_{k}^{\beta}\right)$ \\
\hline $\mathcal{B}_{k}$ & $\begin{array}{l}\text { A new binary training set reconstructed from the original multi-label training set } \\
\mathcal{D} \text { for label } l_{k}\end{array}$ \\
\hline $\operatorname{rank}_{f}(\cdot, \cdot)$ & $\begin{array}{l}\operatorname{rank}_{f}(x, l) \text { returns the rank of } l \text { in } \mathcal{Y} \text { based on the descending order induced from } \\
f(x, \cdot)\end{array}$ \\
\hline
\end{tabular}

different class labels. The first and famous one is LIFT [41], [42]. Others are derived from or inspired by LIFT, e.g., utilizing discriminative features for each Label (ML-DFL) [40], fuzzy rough set (FRSLIFT) [39], meta-label-specific features [32], label-specific features with class-dependent labels in a sparse stacking way (LLSF-DL) [17], selected label-dependent features (SLEF) [26], label-specific features and local pairwise label correlation (LF-LPLC) [38], and performing joint feature selection and classification [18]. In the next section, we will present the LSDM algorithm which handles multi-label data by reconstructing feature space via label specific discriminant mapping features.

\section{THE LSDM ALGORITHM}

Given a training set $\mathcal{D}=\left\{\left(\boldsymbol{x}_{i}, \boldsymbol{y}_{i}\right) \mid 1 \leq i \leq m\right\}$ with $m$ multi-label training examples, where $\boldsymbol{x}_{i} \in \mathcal{X}$ is a $d$-dimensional feature vector and $\boldsymbol{y}_{i} \subseteq \mathcal{Y}$ is the set of relevant labels associated with $\boldsymbol{x}_{i}$. Then, LSDM learns from $\mathcal{D}$ by taking five elementary detailed steps, i.e., label-specific information extraction, distance mapping feature construction, linear representation feature construction, sLDA of reconstructed feature space, and classification model generation.

\subsection{Label-Specific Information Extraction}

The information that could effectively capture the specific characteristics of each label is first extracted, so as to facilitate its discrimination process. It refers to the information from inherent 
properties of the training set with respect to each class label. More specifically, for one class label $l_{k} \in \mathcal{Y}$, we divide the training set into two parts: positive instances set $\mathcal{P}_{k}$ as well as negative instances set $\mathcal{N}_{k}$, which correspond to

$$
\begin{aligned}
& \mathcal{P}_{k}=\left\{\boldsymbol{x}_{i} \mid\left(\boldsymbol{x}_{i}, \boldsymbol{y}_{i}\right) \in \mathcal{D}, l_{k} \in \boldsymbol{y}_{i}\right\}, \\
& \mathcal{N}_{k}=\left\{\boldsymbol{x}_{i} \mid\left(\boldsymbol{x}_{i}, \boldsymbol{y}_{i}\right) \in \mathcal{D}, l_{k} \notin \boldsymbol{y}_{i}\right\} .
\end{aligned}
$$

Intuitively, $\mathcal{P}_{k}$ and $\mathcal{N}_{k}$, defined as label specificity for each label, consist of training instances with and without label $l_{k}$, respectively.

To extract label-specific information from $\mathcal{P}_{k}$ and $\mathcal{N}_{k}$, LSDM chooses to employ partitions of $\mathcal{P}_{k}$ and $\mathcal{N}_{k}$, respectively, as the foundation of reconstructed feature space. Therefore, suppose $\mathcal{P}_{k}$ is partitioned into $m_{k}^{+}$disjoint partitions whose centers are denoted as $C_{k}^{p}=\left\{\boldsymbol{p}_{k}^{1}, \boldsymbol{p}_{k}^{2}, \ldots, \boldsymbol{p}_{k}^{m_{k}^{+}}\right\}$ $\left(C_{k}^{p} \in \mathbb{R}^{d \times m_{k}^{+}}, \boldsymbol{p}_{k} \in \mathbb{R}^{d}\right)$. Similarly, $\mathcal{N}_{k}$ is also partitioned into $m_{k}^{-}$disjoint partitions whose centers are denoted as $C_{k}^{n}=\left\{\boldsymbol{n}_{k}^{1}, \boldsymbol{n}_{k}^{2}, \ldots, \boldsymbol{n}_{k}^{m_{k}^{-}}\right\}\left(C_{k}^{n} \in \mathbb{R}^{d \times m_{k}^{-}}, \boldsymbol{n}_{k} \in \mathbb{R}^{d}\right)$. To determine the appropriate partitions, we consider to optimize the reconstruction error, respectively, defined as

$$
\begin{array}{ll}
\text { minimize } & \sum_{i p=1}^{m_{k}^{p}}\left\|C_{k}^{p} s_{i p}^{p}-x_{i p}^{p}\right\|_{2}^{2} \\
\text { subject to } & \left\|s_{i p}^{p}\right\|_{0,1}=1, \forall i p=1, \ldots, m_{k}^{p}
\end{array}
$$

and

$$
\begin{array}{ll}
\text { minimize } & \sum_{i n=1}^{m_{k}^{n}}\left\|C_{k}^{n} \boldsymbol{s}_{i n}^{n}-\boldsymbol{x}_{i n}^{n}\right\|_{2}^{2} \\
\text { subject to } & \left\|\boldsymbol{s}_{i n}^{n}\right\|_{0,1}=1, \forall i n=1, \ldots, m_{k}^{n},
\end{array}
$$

where $\boldsymbol{s}_{i p}^{p} \in \mathbb{R}^{m_{k}^{+}}, \boldsymbol{s}_{i n}^{n} \in \mathbb{R}^{m_{k}^{-}}, \boldsymbol{x}_{i p}^{p} \in \mathcal{P}_{k}, \boldsymbol{x}_{i n}^{n} \in \mathcal{N}_{k}, m_{k}^{p}=\left|\mathcal{P}_{k}\right|$, and $m_{k}^{n}=\left|\mathcal{N}_{k}\right|$ are the number of positive and negative instances for each class label, respectively. $|\cdot|$ returns the set cardinality.

However, to determine the centers of partitions, it is hard to be optimized due to the condition $\|\boldsymbol{s}\|_{0,1}=1(0-$ norm, $1-$ norm $)$. As a compromise, the popular $k$-means clustering algorithm is employed to handle this [19]. Although it might be suboptimal due to the centers of initialization and the number of iterations, it is effective and simple. Multi-label learning tasks usually suffer from the issue of class imbalance [45], where the number of positive instances for each class label is much smaller than the number of negative ones, i.e., $m_{k}^{p} \ll m_{k}^{n}$. To alleviate the potential risks brought by the class-imbalance problem, LSDM sets equivalent number of clusters for $\mathcal{P}_{k}$ and $\mathcal{N}_{k}$, i.e., $m_{k}^{+}=m_{k}^{-}=m_{k}$. In this way, clustering information gained from positive instances as well as negative instances are treated with equal importance.

Specifically, the number of clusters retained for $\mathcal{P}_{k}$ and $\mathcal{N}_{k}$ is set as follows:

$$
m_{k}=\left\lceil\beta \cdot \min \left(m_{k}^{p}, m_{k}^{n}\right)\right\rceil,
$$

where $\lceil\cdot\rceil$ denotes the retained integer and $\beta \in[0,1]$ is a ratio parameter controlling the number of retained clusters. In this article, we set several values for $\beta$ simultaneously, which is different from LIFT.

\subsection{Distance Mapping}

To exploit the label-specific information represented by the cluster centers with regard to each label, distance mapping features as in LIFT are constructed by using distances between instances and the cluster centers as feature representation. Intuitively, the cluster centers generated by the 
$k$-means algorithm characterize the underlying structure of the original feature space with regard to $l_{k}$, which can be served as appropriate building blocks (prototypes) for the construction of label specific features. Here, a mapping $\phi_{k}^{\prime}: \mathcal{X} \rightarrow \mathcal{Z}_{k}^{\prime}$ from the original $d$-dimensional input space $\mathcal{X}$ to the $2 m_{k}$-dimensional distance mapping feature space is created as follows:

$$
\phi_{k}^{\prime \mathrm{T}}(\boldsymbol{x})=\left[d_{p_{k}}^{1}, \ldots, d_{p_{k}}^{m_{k}}, d_{n_{k}}^{1}, \ldots, d_{n_{k}}^{m_{k}}\right],
$$

where

$$
d_{p_{k}}^{i d}=\left\|\boldsymbol{x}-\boldsymbol{p}_{k}^{i d}\right\|_{2} \text { and } d_{n_{k}}^{i d}=\left\|\boldsymbol{x}-\boldsymbol{n}_{k}^{i d}\right\|_{2} \quad\left(1 \leq i d \leq m_{k}\right) .
$$

Here, we employ the Euclidean distance $(2-$ norm $)$ as the metric in this article.

\subsection{Linear Representation}

We consider that the distance mapping features are not good enough to capture all the labelspecific information, so we employ linear representation features which are using the weights derived from the instances linearly represented by the cluster centers with regard to $l_{k}$ to further exploit such information. The first subset of features mainly contain far and near distance information between instances and the cluster centers, while the second subset of features model the spatial topological information between them. The combination of these two subsets of features can well utilize the label-specific information. Specifically, each instance can be represented as the linear weighted sum of all the positive and negative cluster centers. Here, a mapping $\phi_{k}^{\prime \prime}: \mathcal{X} \rightarrow \mathcal{Z}_{k}^{\prime \prime}$ from the original $d$-dimensional input space $\mathcal{X}$ to the $2 m_{k}$-dimensional linear representation feature space is created as follows:

$$
\phi_{k}^{\prime \prime T}(\boldsymbol{x})=\left[w_{k}^{1}, w_{k}^{2}, \ldots, w_{k}^{2 m_{k}}\right],
$$

where $w_{k}^{j}\left(1 \leq j \leq 2 m_{k}\right)$ is the reconstructed weight for each of the positive and negative cluster centers of each class label and $\boldsymbol{w}_{k}^{\mathrm{T}}=\left\{w_{k}^{1}, w_{k}^{2}, \ldots, w_{k}^{2 m_{k}}\right\}\left(\boldsymbol{w}_{k} \in \mathbb{R}^{2 m_{k}}\right)$. Further for representation consistency, we constrain $\sum_{j=1}^{2 m_{k}} w_{k}^{j}=1$.

Accordingly, the problem above can be defined as a solution problem as follows:

$$
\begin{array}{ll}
\text { minimize } & \sum_{i=1}^{m}\left\|\boldsymbol{x}_{i}-\sum_{j=1}^{2 m_{k}} w_{k i}^{j} C_{k}^{j}\right\|_{2}^{2} \\
\text { subject to } & \sum_{j}^{2 m_{k}} w_{k i}^{j}=1, \forall j=1, \ldots, 2 m_{k},
\end{array}
$$

where $C_{k}=\left[C_{k}^{p}, C_{k}^{n}\right]=\left\{\boldsymbol{p}_{k}^{1}, \ldots, \boldsymbol{p}_{k}^{m_{k}}, \boldsymbol{n}_{k}^{1}, \ldots, \boldsymbol{n}_{k}^{m_{k}}\right\}\left(C_{k} \in \mathbb{R}^{d \times 2 m_{k}}\right)$, and $C_{k}^{j}$ is the $j$ th column of $C_{k}$ for $l_{k}$.

To compute $\boldsymbol{w}_{k i}$, whose computational process is similar to the computation of local linear embedding [29], we can solve a linear equation as follows:

$$
C \mathcal{M}_{k i} \cdot w^{\prime}{ }_{k i}=1
$$

where $\boldsymbol{w}^{\prime}{ }_{k i} \in \mathbb{R}^{2 m_{k}}$ and $\mathbf{1}^{\mathrm{T}}=\{1, \ldots, 1\}\left(\mathbf{1} \in \mathbb{R}^{d}\right) . C \mathcal{M}_{k i}$ is the covariance matrix about $\boldsymbol{x}_{i}$ and $C_{k}$ for each class label $l_{k}$

$$
C \mathcal{M}_{k i}=\left(\begin{array}{ccc}
\left(\boldsymbol{x}_{i}-C_{k}^{1}\right)^{\mathrm{T}}\left(\boldsymbol{x}_{i}-C_{k}^{1}\right) & \cdots & \left(\boldsymbol{x}_{i}-C_{k}^{1}\right)^{\mathrm{T}}\left(\boldsymbol{x}_{i}-C_{k}^{2 m_{k}}\right) \\
\vdots & \ddots & \vdots \\
\left(\boldsymbol{x}_{i}-C_{k}^{2 m_{k}}\right)^{\mathrm{T}}\left(\boldsymbol{x}_{i}-C_{k}^{1}\right) & \cdots & \left(\boldsymbol{x}_{i}-C_{k}^{2 m_{k}}\right)^{\mathrm{T}}\left(\boldsymbol{x}_{i}-C_{k}^{2 m_{k}}\right)
\end{array}\right) \text {. }
$$




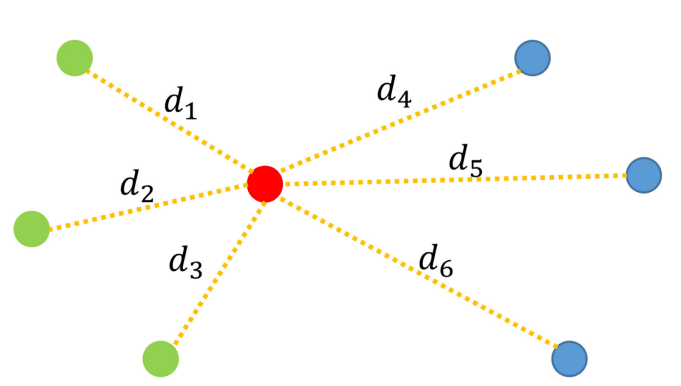

(a) Distance Mapping

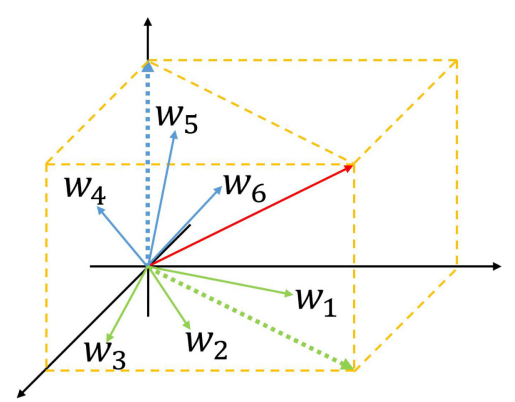

(b) Linear Representation

Fig. 2. Schematics of (a) distance mapping and (b) linear representation. Red point (arrow) represents original instance, green and blue points (arrows) represent the cluster centers from positive and negative instances, respectively.

To compute the $\boldsymbol{w}^{\prime}{ }_{k i}$ and avoid $C \mathcal{M}_{k i}$ is not invertible, we should use the regularization form $C \mathcal{M}_{k i}^{\prime}$, then

$$
\boldsymbol{w}_{k i}^{\prime}=C \mathcal{M}_{k i}^{\prime}{ }^{-1} \cdot \mathbf{1}
$$

At last, we normalize the form $\boldsymbol{w}^{\prime}{ }_{k i}$ to get the reconstructed weights $\boldsymbol{w}_{k_{i}}$ according to Equation (6).

$$
\boldsymbol{w}_{k i}=\frac{\boldsymbol{w}_{k_{i}}}{\sum_{j=1}^{2 m_{k}} \boldsymbol{w}_{k i}^{\prime j}} .
$$

To better understand the concepts of the second and the third steps, Figure 2 illustrates the schematics of distance mapping and linear representation.

\subsection{Simplified Linear Discriminant Analysis}

For $l_{k}$ and $\beta$, a mapping $\phi_{k}: \mathcal{X} \rightarrow \mathcal{Z}_{k}$ from the original $d$-dimensional input space $\mathcal{X}$ to the $4 m_{k}$ dimensional reconstructed feature space is created as follows:

$$
\phi_{k}^{\mathrm{T}}(\boldsymbol{x})=\left[\phi_{k}^{\prime T}(\boldsymbol{x}), \phi_{k}^{\prime \prime T}(\boldsymbol{x})\right],
$$

where $\phi_{k}(\boldsymbol{x})$ is the label-specific features for label $l_{k}$ and instance $\boldsymbol{x}$ which is the coalition of distance mapping and linear representation features.

Specifically, about parameter sensitivity, for each identical class label $l_{k}$, each $\beta$ value corresponds to proper feature mapping $\phi_{k}^{\beta}$. Hence, for one class label $l_{k} \in \mathcal{Y}$ and $\beta$, we reconstruct the training set into two parts: positive instances set $\mathcal{P}_{k}^{\beta}$ as well as negative instances set $\mathcal{N}_{k}^{\beta}$, which correspond to

$$
\begin{aligned}
& \mathcal{P}_{k}^{\beta}=\left\{\phi_{k}^{\beta}\left(\boldsymbol{x}_{i}\right) \mid\left(\phi_{k}^{\beta}\left(\boldsymbol{x}_{i}\right), \boldsymbol{y}_{i}\right) \in \mathcal{D}, l_{k} \in \boldsymbol{y}_{i}\right\}, \\
& \mathcal{N}_{k}^{\beta}=\left\{\phi_{k}^{\beta}\left(\boldsymbol{x}_{i}\right) \mid\left(\phi_{k}^{\beta}\left(\boldsymbol{x}_{i}\right), \boldsymbol{y}_{i}\right) \in \mathcal{D}, l_{k} \notin \boldsymbol{y}_{i}\right\} .
\end{aligned}
$$

To excavate an appropriate $\phi_{k}$ well predicting unseen instances, one intuitive strategy is to exploit diverse $\beta$ values to generate multiple mappings, and then train classifiers with regard to each $\phi_{k}^{\beta}$ and test them to discriminate optimal $\phi_{k}$. However, when the number of ratio value and size of dataset are large, this strategy faces the dilemma that training too many classifiers increases computational complexity and time also.

We assume that if a reconstructed feature space for label $l_{k}$ is good, the distances for instances within class are small and those between classes are large. Here, class means positive or negative instances for label $l_{k}$. Due to this assumption, we propose a SLDA, which only computes two 
mean values and scatters of positive and negative training instances based on reconstructed feature space for each label without mapping to one-dimension space (dimension reduction), in order to deal with it efficiently as a compromise. sLDA is employed to achieve highly efficient excavation of optimal feature space from different reconstructed feature spaces of identical class label. Intuitively, a mapping which can separate the instances belonging to $l_{k}$ or not as well as possible is good. Specifically, $\boldsymbol{m}_{p k}^{\beta}$ and $\boldsymbol{m}_{n k}^{\beta}$ are the feature vectors' mean value of $\mathcal{P}_{k}^{\beta}$ and $\mathcal{N}_{k}^{\beta}$, respectively, denoted as follows:

$$
\begin{aligned}
\boldsymbol{m}_{p k}^{\beta} & =\frac{\sum_{i=1}^{m} \phi_{k}^{\beta}\left(\boldsymbol{x}_{i}\right) \delta_{i}^{\beta}}{\sum_{i=1}^{m} \delta_{i}^{\beta}}, \\
\boldsymbol{m}_{n k}^{\beta} & =\frac{\sum_{i=1}^{m} \phi_{k}^{\beta}\left(\boldsymbol{x}_{i}\right)\left(1-\delta_{i}^{\beta}\right)}{\sum_{i=1}^{m}\left(1-\delta_{i}^{\beta}\right)},
\end{aligned}
$$

where $\delta_{i}^{\beta}=1$ with regard to $\phi_{k}^{\beta}\left(\boldsymbol{x}_{i}\right) \in \mathcal{P}_{k}^{\beta}$ and $\delta_{i}^{\beta}=0$ with regard to $\phi_{k}^{\beta}\left(\boldsymbol{x}_{i}\right) \in \mathcal{N}_{k}^{\beta}$. And the scatters, indicated as $s_{p k}^{\beta}$ and $s_{n k}^{\beta}$, of $\mathcal{P}_{k}^{\beta}$ and $\mathcal{N}_{k}^{\beta}$ are defined as follows:

$$
\begin{aligned}
& s_{p k}^{\beta}=\sum_{i=1}^{m}\left\|\phi_{k}^{\beta}\left(\boldsymbol{x}_{i}\right)-\boldsymbol{m}_{p k}^{\beta}\right\|_{2}^{2} \delta_{i}^{\beta}, \\
& s_{n k}^{\beta}=\sum_{i=1}^{m}\left\|\phi_{k}^{\beta}\left(\boldsymbol{x}_{i}\right)-\boldsymbol{m}_{n k}^{\beta}\right\|_{2}^{2}\left(1-\delta_{i}^{\beta}\right) .
\end{aligned}
$$

To alleviate the potential risks brought by the class-imbalance problem, we average the scatters as follows:

$$
\begin{aligned}
& \overline{s_{p k}^{\beta}}=\frac{\sum_{i=1}^{m}\left\|\phi_{k}^{\beta}\left(\boldsymbol{x}_{i}\right)-\boldsymbol{m}_{p k}^{\beta}\right\|_{2}^{2} \delta_{i}^{\beta}}{\sum_{i=1}^{m} \delta_{i}^{\beta}}, \\
& \overline{s_{n k}^{\beta}}=\frac{\sum_{i=1}^{m}\left\|\phi_{k}^{\beta}\left(\boldsymbol{x}_{i}\right)-\boldsymbol{m}_{n k}^{\beta}\right\|_{2}^{2}\left(1-\delta_{i}^{\beta}\right)}{\sum_{i=1}^{m}\left(1-\delta_{i}^{\beta}\right)} .
\end{aligned}
$$

After mapping, for well separating the instances belonging to $l_{k}$ or not with regard to each $\beta$, we hope the distance between $\boldsymbol{m}_{p k}^{\beta}$ and $\boldsymbol{m}_{n k}^{\beta}$ is as far as possible and the scatters of $\mathcal{P}_{k}^{\beta}$ and $\mathcal{N}_{k}^{\beta}$ are as small as possible. Hence, we employ sLDA to denote this as follows:

$$
J\left(\phi_{k}^{\beta}\right)=\frac{\left\|\boldsymbol{m}_{p k}^{\beta}-\boldsymbol{m}_{n k}^{\beta}\right\|_{2}^{2}}{\overline{s_{p k}^{\beta}}+\overline{s_{n k}^{\beta}}},
$$

where $J\left(\phi_{k}^{\beta}\right)$ denotes the performance of each $\beta$ with regard to $l_{k}$. We excavate the optimal $\phi_{k}$ due to the largest $J\left(\phi_{k}^{\beta}\right)$.

\subsection{Classification Model Generation}

A family of $q$ classifiers $\left\{\mathfrak{c}_{1}, \mathfrak{c}_{2}, \ldots, \mathfrak{c}_{q}\right\}$ are induced with the generated label-specific discriminant mapping features. Here, for each class label $l_{k} \in \mathcal{Y}$, a new binary training set $\mathcal{B}_{k}$ with $m$ examples is reconstructed from the original multi-label training set $\mathcal{D}$ and the optimal mapping $\phi_{k}$ with regard to largest $J\left(\phi_{k}^{\beta}\right)$ as follows:

$$
\mathcal{B}_{k}=\left\{\left(\phi_{k}\left(\boldsymbol{x}_{i}\right), \boldsymbol{y}_{i}(k) \mid\left(\boldsymbol{x}_{i}, \boldsymbol{y}_{i}\right) \in \mathcal{D}\right)\right\} \text {. }
$$


Here, $\boldsymbol{y}_{i}(k)=+1$ if $l_{k} \in \boldsymbol{y}_{i}$; otherwise, $\boldsymbol{y}_{i}(k)=-1$. Based on $\mathcal{B}_{k}$, any binary learner $\mathfrak{Q}$ can be applied to induce a classifier $\mathfrak{c}_{k}: \mathcal{Z}_{k} \rightarrow \mathbb{R}$ for $l_{k}$. The detailed discussion about the binary learner is described in Experiments part.

Give an unseen instance $\boldsymbol{u} \in \mathcal{X}$, its associated label set is predicted as

$$
\boldsymbol{y}=\left\{l_{k} \mid \mathfrak{c}_{k}\left(\phi_{k}(\boldsymbol{u})\right)>0,1 \leq k \leq q\right\} .
$$

In other words, classification model $f_{k}$ corresponding to each label $l_{k}$ can be viewed as the composition of $\mathfrak{c}_{k}$ and $\phi_{k}$, i.e., $f_{k}(u)=\left[\mathfrak{c}_{k} \circ \phi_{k}\right](\boldsymbol{u})=\mathfrak{c}_{k}\left(\phi_{k}(\boldsymbol{u})\right)$. Algorithm 1 illustrates the procedure of LSDM in detail.

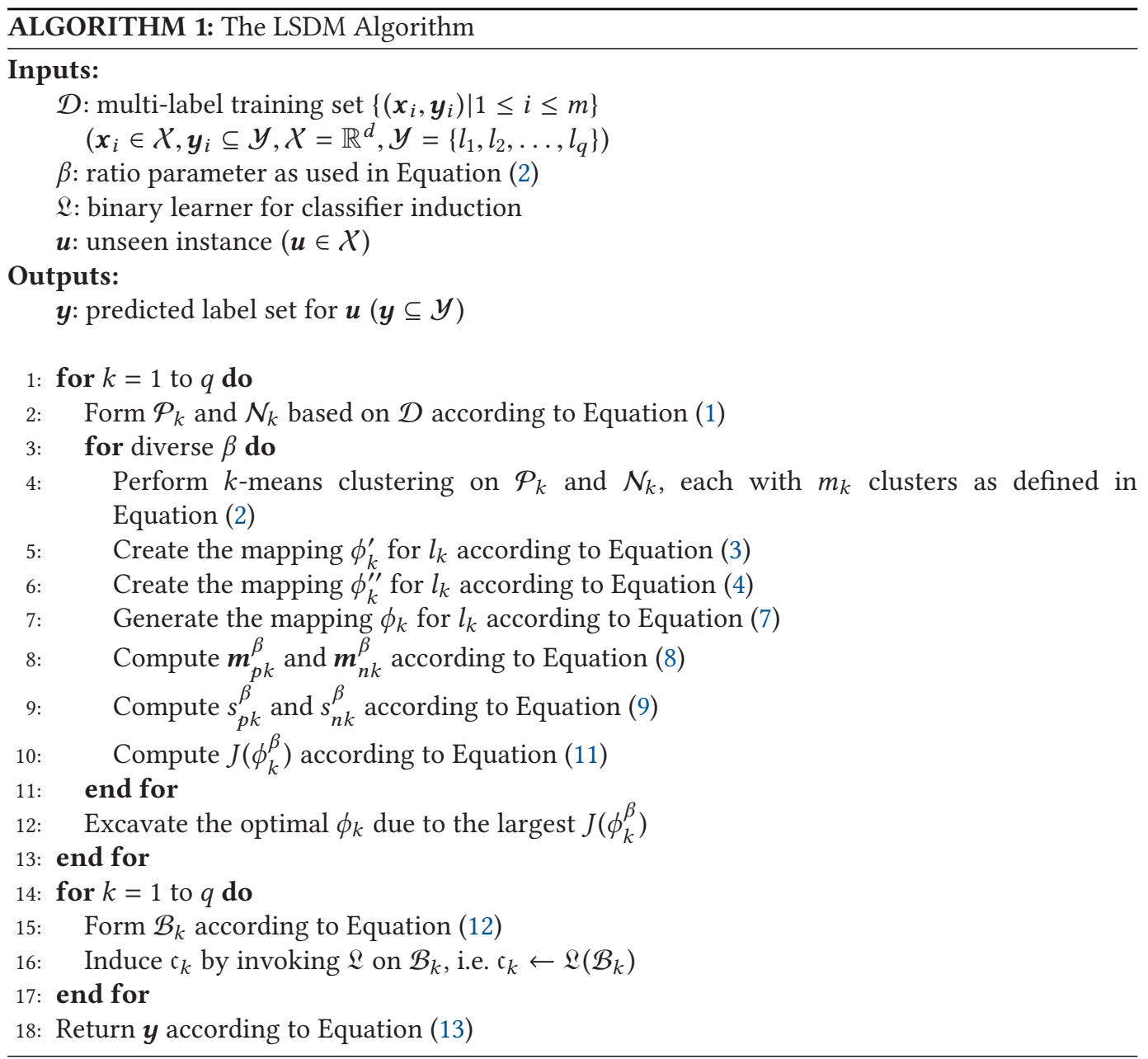

\section{EXPERIMENTS}

\subsection{Datasets}

For each dataset, $|S|, \operatorname{dim}(S), L(S)$, and $F(S)$ denote the number of examples, number of features, number of possible class labels, and feature type for $S$, respectively. In addition, several other multilabel properties [35], [28] are further used, including label cardinality LCard $(S)$ which measures 
the average number of labels per example, label density LDen $(S)$ which normalizes LCard $(S)$ by the number of possible labels, distinct label sets $\mathrm{DL}(S)$ which counts the number of distinct label combinations in $S$, proportion of distinct label sets $\operatorname{PDL}(S)$ which normalizes $\operatorname{DL}(S)$ by the number of example. They are denoted as

$$
\begin{aligned}
& \operatorname{LCard}(S)=\frac{1}{p} \sum_{i=1}^{p}\left|\boldsymbol{y}_{i}\right|, \\
& \operatorname{LDen}(S)=\frac{\operatorname{LCard}(S)}{L(S)}, \\
& \operatorname{DL}(S)=|\{Y \mid(x, Y) \in S\}|, \\
& \operatorname{PDL}(S)=\frac{\operatorname{DL}(S)}{|S|} .
\end{aligned}
$$

In summary, detailed characteristics of all multi-label datasets used in the experiments are demonstrated in Table 2. Roughly ordered by $|S|, 10$ regular-scale datasets (first part, $|S|<5,000$ ) as well as 10 large-scale datasets (second part, $|S| \geq 5,000$ ) are included. Furthermore, dimensionality reduction is performed on three text datasets with huge number of features $(\operatorname{dim}(S)>47,000)$, including $r c v 1$ (subset 1), $r c v 1$ (subset 2), and tmc2007. Specifically, the top $2 \%$ features with the highest document frequency are retained. These datasets cover a broad range of cases with diversified multi-label properties. Therefore, experimental studies reported in this article are quite comprehensive to provide a solid basis for thorough evaluation of LSDM's effectiveness.

\subsection{Evaluation Measures}

In the multi-label learning community, it is well known that the performance evaluation of multilabel learning differs from that of classical single-label learning because each example could have multiple labels simultaneously. Following the notations in Table 1, six standard evaluation measures are introduced for evaluating the performance of our proposed method from multiple aspects, namely, Average precision, Macro-averaging AUC, Hamming loss, Coverage, One-error, and Ranking loss [35], [45]. They are defined as follows:

- Average precision:

$$
\text { avgprec }=\frac{1}{t} \sum_{i=1}^{t} \frac{1}{\left|Y_{i}\right|} \sum_{l_{k} \in Y_{i}} \frac{\left|\mathcal{R}\left(\boldsymbol{x}_{i}, l_{k}\right)\right|}{\operatorname{rank}_{f}\left(\boldsymbol{x}_{i}, l_{k}\right)},
$$

where

$$
\mathcal{R}\left(\boldsymbol{x}_{i}, l_{k}\right)=\left\{l_{j} \mid \operatorname{rank}_{f}\left(\boldsymbol{x}_{i}, l_{j}\right), l_{j} \in Y_{i}\right\} .
$$

Here, $\operatorname{rank}_{f}\left(\boldsymbol{x}_{i}, l_{k}\right)=\sum_{j=1}^{q}\left\|f_{j}\left(\boldsymbol{x}_{i}\right) \geq f_{k}\left(\boldsymbol{x}_{i}\right)\right\|$ returns the rank of $l_{k}$ when all class labels in $\boldsymbol{Y}$ are sorted in descending order according to $\left\{f_{1}\left(\boldsymbol{x}_{i}\right), f_{2}\left(\boldsymbol{x}_{i}\right), \ldots, f_{q}\left(\boldsymbol{x}_{i}\right)\right\}$. Average precision evaluates the average fraction of relevant labels ranked higher than a particular label $l_{k} \in Y_{i}$. - Macro-averaging AUC:

$$
\begin{gathered}
\mathrm{AUC}_{\text {macro }}=\frac{1}{q} \sum_{k=1}^{q} \mathrm{AUC}_{k} \\
=\frac{1}{q} \sum_{k=1}^{q} \frac{\left|\left\{\left(\boldsymbol{x}^{\prime}, \boldsymbol{x}^{\prime \prime}\right) \mid f_{k}(\boldsymbol{x}) \geq f_{k}(\boldsymbol{x}),\left(\boldsymbol{x}^{\prime}, \boldsymbol{x}^{\prime \prime}\right) \in \mathcal{P}_{k} \times \mathcal{N}_{k}\right\}\right|}{m_{k}^{p} m_{k}^{n}},
\end{gathered}
$$

Here, the AUC value on each class label (i.e., $\mathrm{AUC}_{k}$ ) is calculated based on the relationship between AUC and the Wilcoxon-Mann-Whitney statistic [14]. 
Table 2. Characteristics of Datasets

\begin{tabular}{|c|c|c|c|c|c|c|c|c|c|c|}
\hline Dataset & $|\mathcal{S}|$ & $\operatorname{dim}(\mathcal{S})$ & $L(\mathcal{S})$ & $F(\mathcal{S})$ & $\operatorname{LCard}(\mathcal{S})$ & $\operatorname{LDen}(S)$ & $\mathrm{DL}(\mathcal{S})$ & $\operatorname{PDL}(\mathcal{S})$ & Domain & $\mathrm{URL}^{\star}$ \\
\hline CAL500 & 502 & 68 & 174 & Numeric & 26.044 & 0.150 & 502 & 1.000 & Music & URL 1 \\
\hline Emotions & 593 & 72 & 6 & Numeric & 1.869 & 0.311 & 27 & 0.046 & Music & URL 1 \\
\hline Genbase & 662 & 1,185 & 27 & Nominal & 1.252 & 0.046 & 32 & 0.048 & Biology & URL 1 \\
\hline Medical & 978 & 1,449 & 45 & Nominal & 1.245 & 0.028 & 94 & 0.096 & Text & URL 2 \\
\hline Language log & 1,460 & 1,004 & 75 & Nominal & 1.180 & 0.016 & 286 & 0.196 & Text & URL 2 \\
\hline Enron & 1,702 & 1,001 & 53 & Nominal & 3.378 & 0.064 & 753 & 0.442 & Text & URL 2 \\
\hline Image & 2,000 & 294 & 5 & Numeric & 1.236 & 0.247 & 20 & 0.010 & Images & URL 3 \\
\hline Scene & 2,407 & 294 & 6 & Numeric & 1.074 & 0.179 & 15 & 0.006 & Images & URL 1 \\
\hline Yeast & 2,417 & 103 & 14 & Numeric & 4.237 & 0.303 & 198 & 0.082 & Biology & URL 3 \\
\hline Slashdot & 3,782 & 1,079 & 22 & Nominal & 1.181 & 0.054 & 156 & 0.041 & Text & URL 2 \\
\hline Corel5k & 5,000 & 499 & 374 & Nominal & 3.522 & 0.009 & 3,175 & 0.635 & Images & URL 1 \\
\hline rcv1(subset1) & 6,000 & 944 & 101 & Numeric & 2.880 & 0.029 & 1,028 & 0.171 & Text & URL 1 \\
\hline rcv1(subset2) & 6,000 & 944 & 101 & Numeric & 2.634 & 0.026 & 954 & 0.159 & Text & URL 1 \\
\hline bibtex & 7,395 & 1,836 & 159 & Nominal & 2.402 & 0.015 & 2,856 & 0.386 & Text & URL 1 \\
\hline Corel16k(sample1) & 13,766 & 500 & 153 & Nominal & 2.859 & 0.019 & 4,803 & 0.349 & Images & URL 1 \\
\hline Corel16k(sample2) & 13,761 & 500 & 164 & Nominal & 2.882 & 0.018 & 4,868 & 0.354 & Images & URL 1 \\
\hline Eurlex(suject matter) & 19,348 & 5,000 & 201 & Numeric & 2.213 & 0.011 & 2,504 & 0.129 & Text & URL 1 \\
\hline Eurlex(directory code) & 19,348 & 5,000 & 412 & Numeric & 1.292 & 0.003 & 1,615 & 0.084 & Text & URL 1 \\
\hline tmc2007 & 28,596 & 981 & 22 & Nominal & 2.158 & 0.098 & 1,341 & 0.047 & Text & URL 1 \\
\hline mediamill & 43,907 & 120 & 101 & Numeric & 4.376 & 0.043 & 6,555 & 0.149 & Video & URL 1 \\
\hline
\end{tabular}

^ URL 1: http://mulan.sourceforge.net/datasets.html.

URL 2: http://meka.sourceforge.net/\#datasets.

URL 3: http://cse.seu.edu.cn/people/zhangml/Resources.htm\#data.

-Hamming loss:

$$
\text { hloss }=\frac{1}{t} \sum_{i=1}^{t}\left|h\left(x_{i}\right) \Delta Y_{i}\right| .
$$

Here, $h\left(\boldsymbol{x}_{i}\right)=\left\{l_{k} \mid f_{k}\left(\boldsymbol{x}_{i}\right)>0,1 \leq k \leq q\right\}$ corresponds to the predicted set of relevant labels for $\boldsymbol{x}_{i}$, and $\Delta$ stands for the symmetric difference between two sets. Hamming loss evaluates the fraction of instance-label pairs which have been misclassified, i.e., a relevant label is missed or an irrelevant label is predicted.

- Coverage:

$$
\text { coverage }=\frac{1}{q}\left(\frac{1}{t} \sum_{i=1}^{t} \max \operatorname{rank}_{f}\left(\boldsymbol{x}_{i}, l_{k}\right)-1\right) .
$$

Coverage evaluates how many steps are needed, on average, to move down the ranked label list of an example so as to cover all its relevant labels. Furthermore, the coverage measure is normalized by the number of possible class labels (i.e., $q$ ) in this article.

-One-error:

$$
\text { one-error }=\frac{1}{t} \sum_{t=1}^{t} \|\left[\left[\arg \max _{l_{k} \in \mathcal{Y}} f_{k}\left(\boldsymbol{x}_{i}\right)\right] \notin Y_{i} \| .\right.
$$

Here, for any predicate $\pi,\|\pi\|$ returns 1 if $\pi$ holds and 0 otherwise. One-error evaluates the fraction of examples whose top-ranked predicted label is not in the ground-truth relevant label set. 
- Ranking loss:

$$
\text { rloss }=\frac{1}{t} \sum_{i=1}^{t} \frac{\left|\left\{\left(l_{k}, l_{j}\right) \mid f_{k}\left(\boldsymbol{x}_{i}\right) \leq f_{j}\left(\boldsymbol{x}_{i}\right),\left(l_{k}, l_{j}\right) \in Y_{i} \times \bar{Y}_{i}\right\}\right|}{\left|Y_{i}\right|\left|\bar{Y}_{i}\right|} .
$$

Here, $\bar{Y}_{i}$ is the complementary set of $Y_{i}$ in $\mathcal{Y}$. Ranking loss evaluates the average fraction of misordered label pairs, i.e., an irrelevant label of an example is ranked higher than its relevant one.

Note that for all the six multi-label evaluation measures, their values vary between $[0,1]$. Furthermore, for average precision and macro-averaging AUC, the larger the values the better the performance. While for the other four measures, the smaller the values the better the performance. These measures serve as good indicators for comprehensive comparative studies as they evaluate the performance of the learned models from various aspects.

\subsection{Multi-Label Classifiers}

We compare our proposed algorithm with the following five methods: (1) Label-specific features (LIFT) [41], [42]; (2) BR [2]; (3) Multi-label k nearest neighbors (MLkNN) [44]; (4) Classifier chain (CC) and (5) Ensemble of classifier chains (ECC) [28].

-LIFT [3]: The basic idea of this algorithm is that it first utilizes cluster analysis on the positive and negative instances to construct features specific to each label, and then performs training and testing as $q$ independent binary classification problem by querying the clustering results. It could be viewed as a degenerated version of LSDM where the label specific discriminant mapping features $\phi_{k}(\boldsymbol{x})$ is only kept to the distance mapping features $\phi_{k}^{\prime}(\boldsymbol{x})$ and sLDA is not employed to excavate the optimally reconstructed feature space.

-BR: The basic idea of this algorithm is to decompose the multi-label learning problem into $q$ independent binary classification problems, where each binary classification problem corresponds to a possible label in the label space. It could be viewed as a plain version of LSDM where the feature space is kept to the original features $x$.

- MLkNN. The basic idea of this algorithm is adapting $k$ nearest neighbors techniques to deal with multi-label data, where maximum a posteriori rule is utilized to make prediction by reasoning with the labeling information embodied in the neighbors.

- CC: The basic idea of this algorithm is to transform the multi-label learning problem into a chain of binary classification problems, where subsequent binary classifiers in the chain are successively built upon the predictions of preceding ones.

-ECC: The basic idea of this algorithm is to employ ensemble learning to address chain order randomness as an ensemble of classifier chains. Here, the ensemble size is set to be 50 to accommodate sufficient number of classifier chains.

\subsection{Experimental Setup}

The parameter of LSDM, i.e., ratio $\beta$ as used in Equation (2) is set to be from 0.01 to $0.2(0.01$, $0.05,0.1,0.2)$ in this article. As we mentioned above, our proposed algorithm LSDM can utilize all these $\beta$ values at the same time and employ sLDA to efficiently excavate the optimal $\phi_{k}$. This is different from LIFT, because it can only utilize one $\beta$ value at a time. The parameter settings of LIFT, MLkNN, and ECC are as suggested in the corresponding literatures. The ratio of LIFT is set to be 0.1 . The number of nearest neighbors of MLkNN is set to be 10 . The ensemble size and sampling ratio of ECC are set to be 50 and $50 \%$, respectively. For fair comparison, LSDM, LIFT, BR, CC, and ECC employ LIBSVM (with linear kernel) [6] as the binary learner. For the regular-scale 
datasets, we apply 10-fold cross validation. However, for the large-scale datasets, on each dataset, $50 \%$ examples are randomly sampled to form the training set, and the rest are used to form the test set. And then, the sampling process is repeated for 10 times.

\section{RESULTS}

Tables 3 and 4 report the detailed experimental results of all comparing algorithms with six evaluation measures on the regular-scale and large-scale datasets, respectively. For each evaluation measure, " $\uparrow$ " indicates "the larger the better," while " $\downarrow$ " indicates "the smaller the better." Furthermore, the bold-faced values represent the best performance among the six comparing algorithms.

To analyze the performance among the comparing algorithms systematically, we employ Friedman test [10] which is regarded as the favorable statistical test for comparisons among multiple algorithms over a number of datasets. Given $k$ comparing algorithms and $N$ datasets, let $r_{i}^{j}$ denote the rank of the $j$ th algorithm on the $i$ th dataset (mean ranks are shared in case of ties). Let $R_{j}=\frac{1}{N} \sum_{i=1}^{N} r_{i}^{j}$ denote the average rank for the $j$ th algorithm, under the null hypothesis (i.e., all algorithms have "equal" performance), the following Friedman statistic $F_{F}$ will be distributed according to the $F$-distribution with $k-1$ numerator degrees of freedom and $(k-1)(N-1)$ denominator degrees of freedom:

$$
F_{F}=\frac{(N-1) \chi_{F}^{2}}{N(k-1)-\chi_{F}^{2}},
$$

where

$$
\chi_{F}^{2}=\frac{12 N}{k(k+1)}\left[\sum_{j=1}^{k} R_{j}^{2}-\frac{k(k+1)^{2}}{4}\right] .
$$

Table 5 summarizes the Friedman statistics $F_{F}$ and the corresponding critical values on each evaluation measure. At significance level $\alpha=0.05$, the null hypothesis of "equal" performance among the comparing algorithms is clearly rejected on each evaluation measure. Consequently, Bonferroni-Dunn test [11] is employed as the post-hoc test [10] to demonstrate the relative performance among the comparing algorithms, where LSDM is regarded as the control algorithm. Here, the average rank difference between LSDM and one comparing algorithm is compared with the CD:

$$
\mathrm{CD}=q_{\alpha} \sqrt{\frac{k(k+1)}{6 N}}
$$

For Bonferroni-Dunn test, we have $q_{\alpha}=2.576$ at significance level $\alpha=0.05$ and thus CD $=1.524$ $(k=6, N=20)$. Accordingly, the performance between LSDM and one comparing algorithm is deemed to be significantly different if their average ranks differ by at least one CD. Figure 3 illustrates the CD diagrams [11] on each evaluation measure, where the average rank of each comparing algorithm is marked along the axis (lower ranks to the right). In each subfigure, any comparing algorithm whose average rank is within one CD to that of LSDM is interconnected to each other with a thick line. Otherwise, it is considered to have significant different performance against LSDM. Furthermore, Figure 4 illustrates the rank performance of each algorithm corresponding to all datasets on each evaluation measure. In each subfigure, each dataset connects each algorithm with different color curves (performance ranking) simultaneously and the area of different color curves combination for each algorithm on the left half circle denotes its final performance ranking corresponding to all datasets. For example, for subfigure (a) Average precision, we can see that LSDM with area of 20 blue curves (first ranking) combination on the left half circle, which means it ranks first for all datasets. For LIFT, it is with area of 19 celadon curves (second ranking) and 1 blue curve (first ranking). For BR, it is with area of seven orange curves (third ranking), 10 pink 


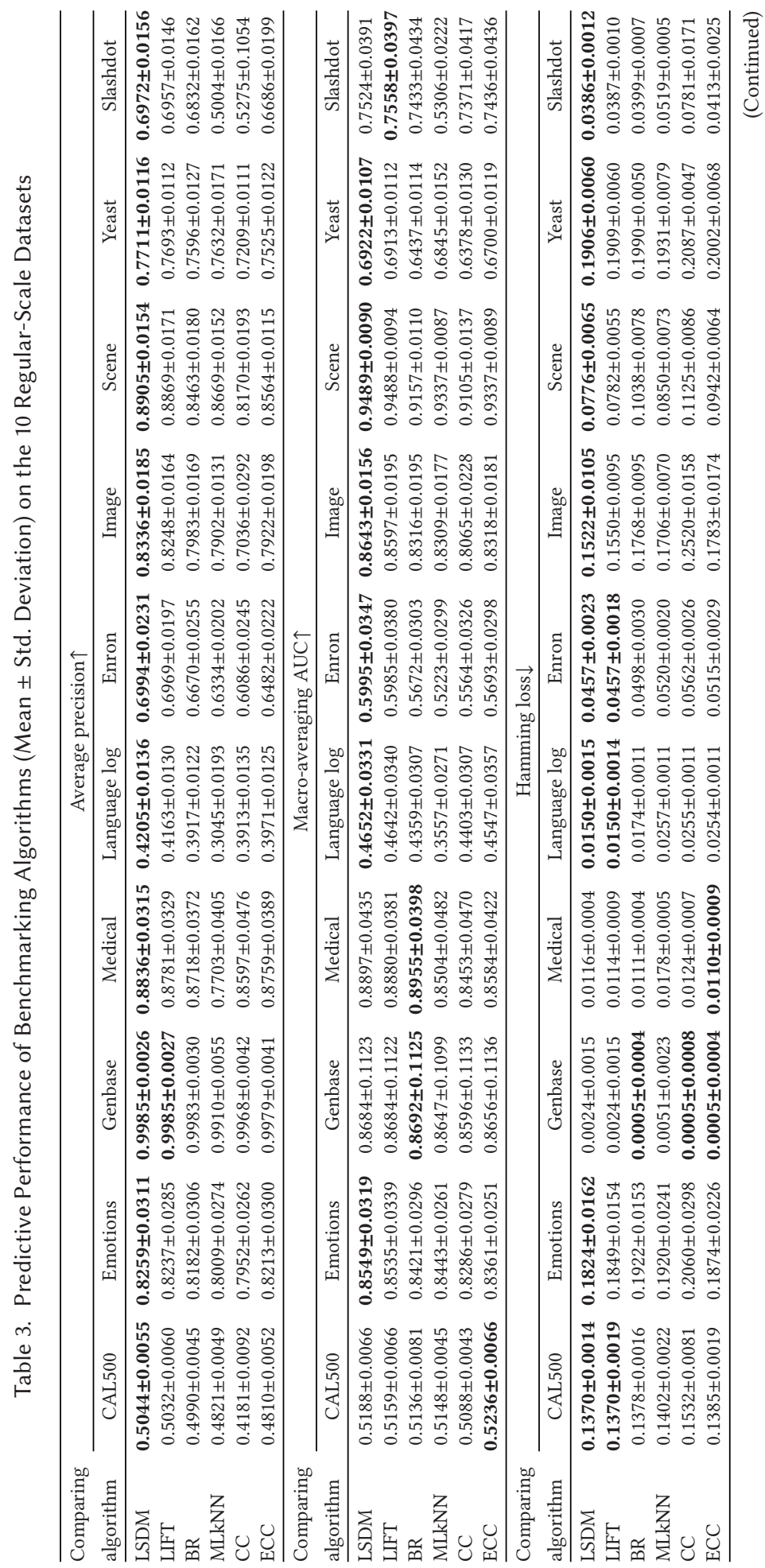




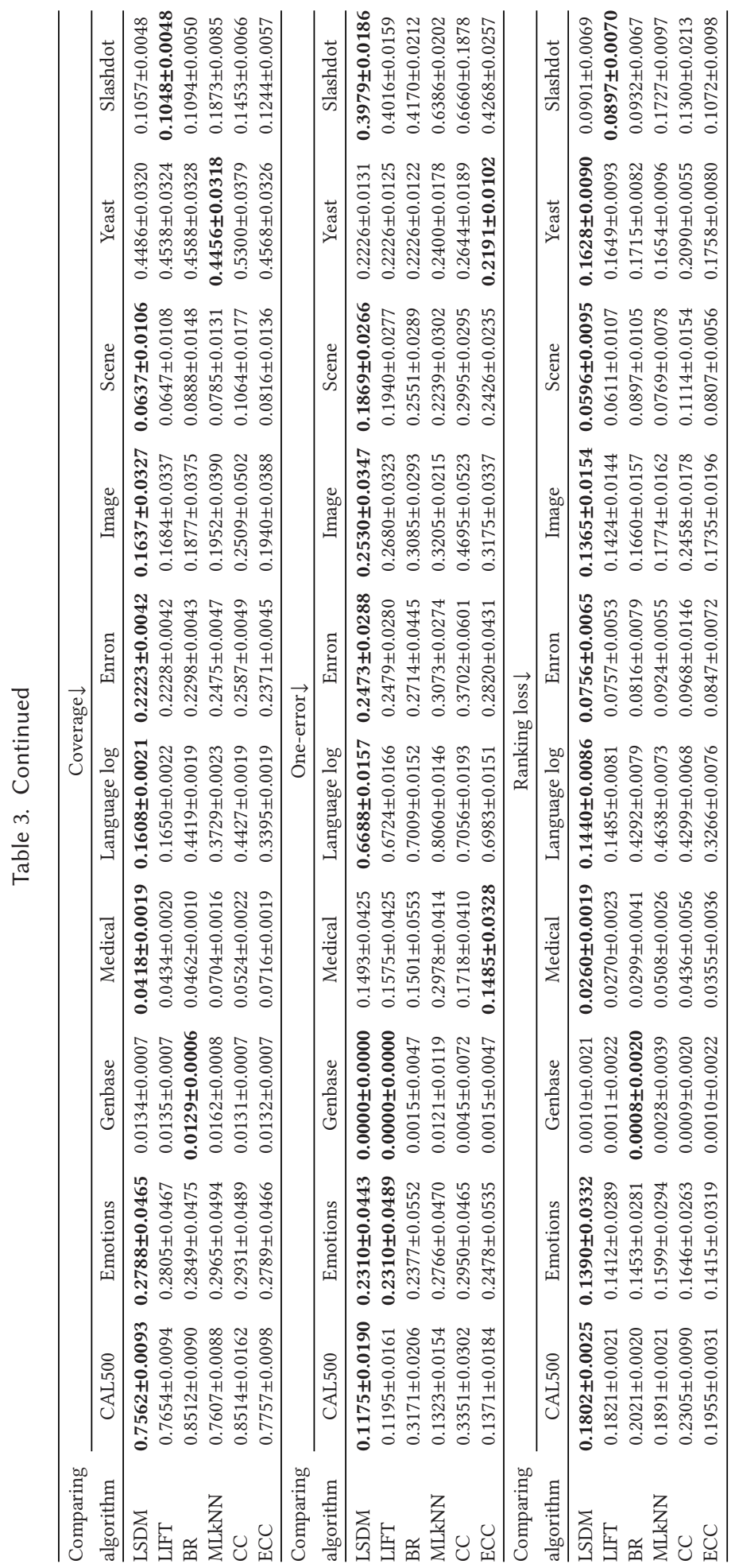




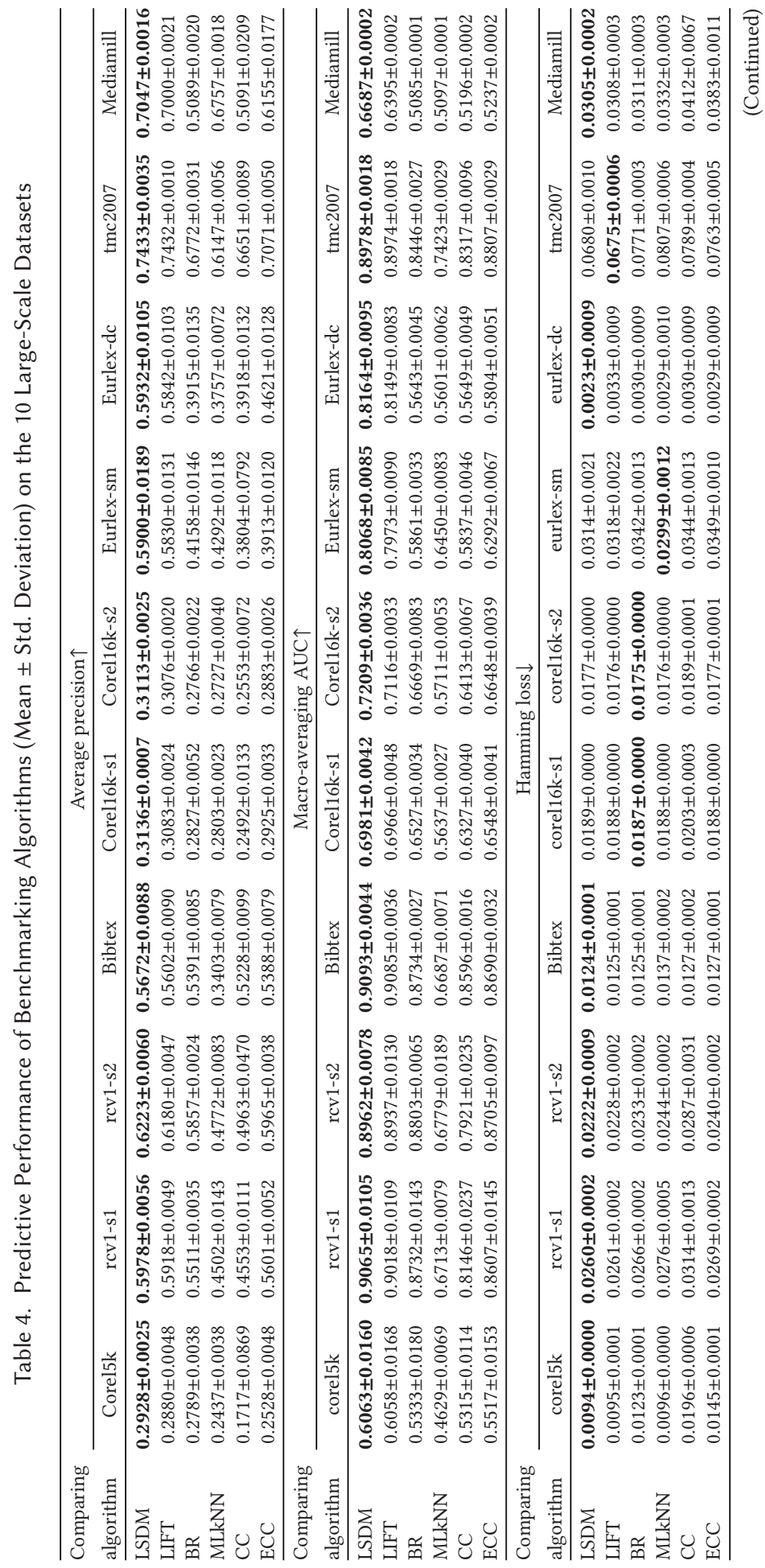




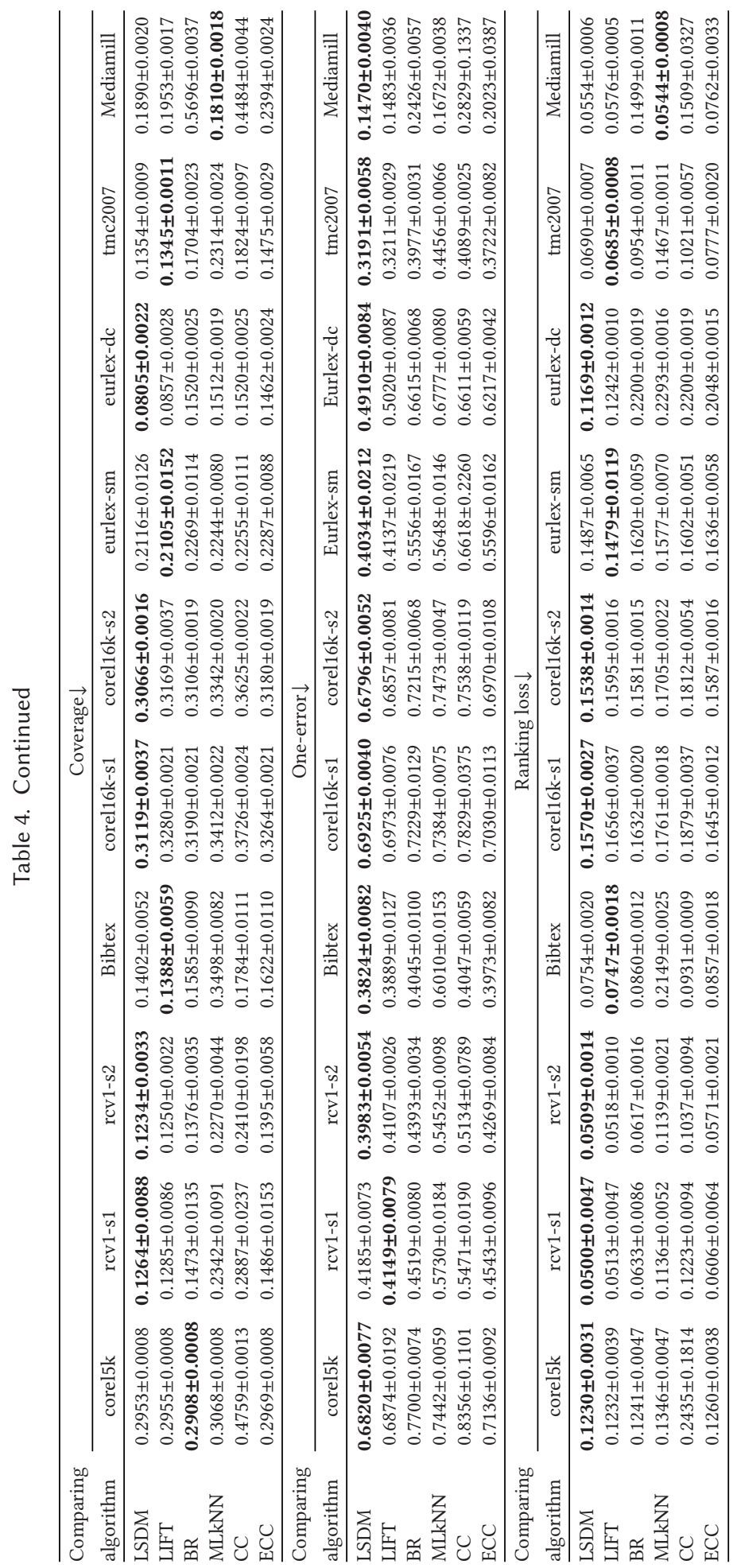


Table 5. Summary of the Friedman Statistics $F_{F}$ in Terms of Each Evaluation Measure and the Critical Value (\# Comparing Algorithms $k=6$; \# Datasets $N=20$ )

\begin{tabular}{lcc}
\hline Evaluation measure & $F_{F}$ & critical value $(\alpha=0.05)$ \\
\hline Average precision & 98.3357 & \\
Macro-averaging AUC & 56.1412 & \\
Hamming loss & 17.4334 & 2.3102 \\
Converage & 23.0420 & \\
One-error & 74.0395 & \\
Ranking loss & 34.3922 & \\
\hline
\end{tabular}

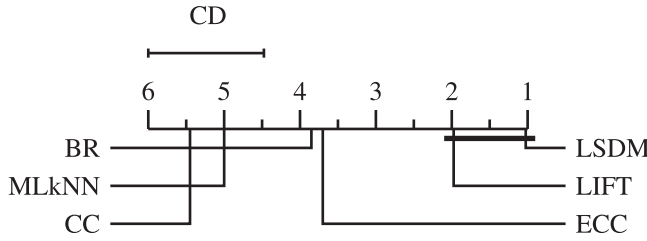

(a) Average precision

$\mathrm{CD}$

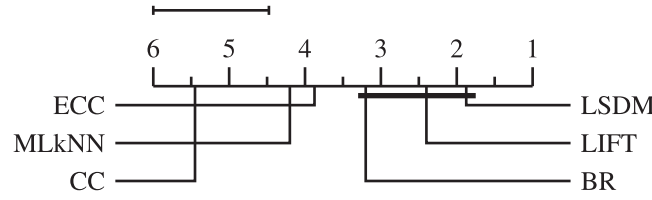

(c) Hamming loss

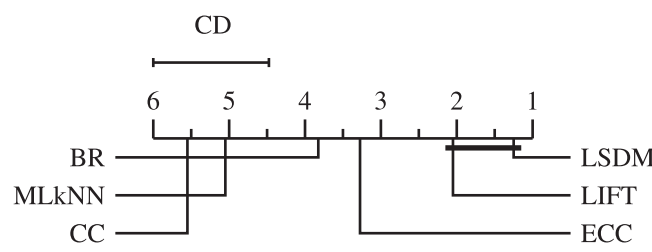

(e) One-error

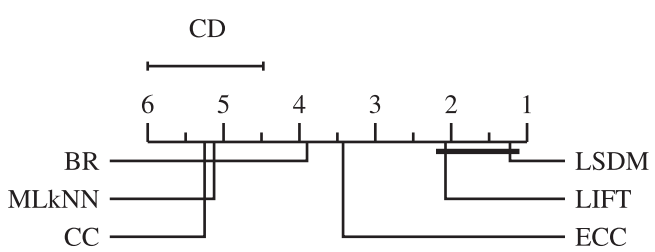

(b) Macro-averaging AUC

$\mathrm{CD}$

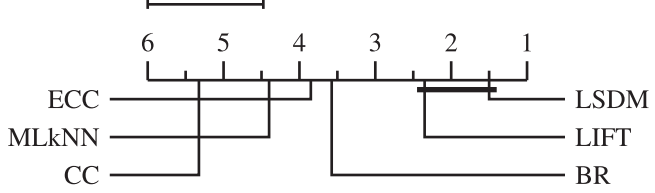

(d) Coverage

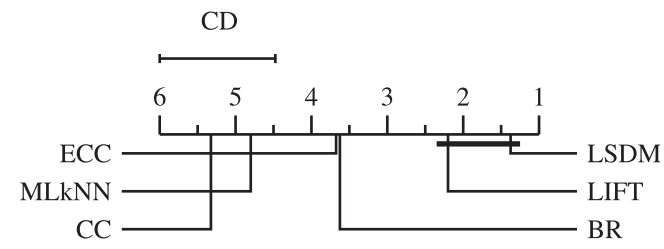

(f) Ranking loss

Fig. 3. Comparison of LSDM (control algorithm) against other comparing algorithms with Bonferroni-Dunn test under each evaluation measure. Algorithms not connected with LSDM in the CD diagram are considered to have significantly different performance from the control algorithm (significance level $\alpha=0.05$ ).

curves (fourth ranking), 2 green curves (fifth ranking), and 1 purple curve (sixth ranking). The other three are described in the same way.

Based on the above experimental results, the following observations can be apparently made:

(1) As shown in Figure 3, the performance in terms of each evaluation measure between LSDM and LIFT is not significantly different due to their average ranks within least one $\mathrm{CD}$, but we can also consider that LSDM achieves comparable performance against LIFT. Because the Bonferroni-Dunn test measures the average rank and LIFT is excellent to beat 


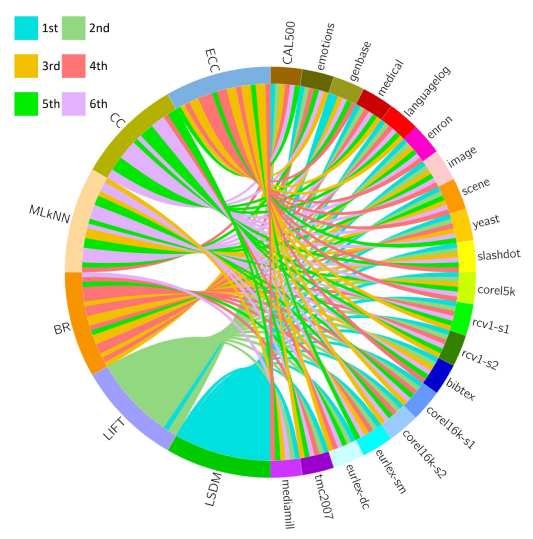

(a) Average precision

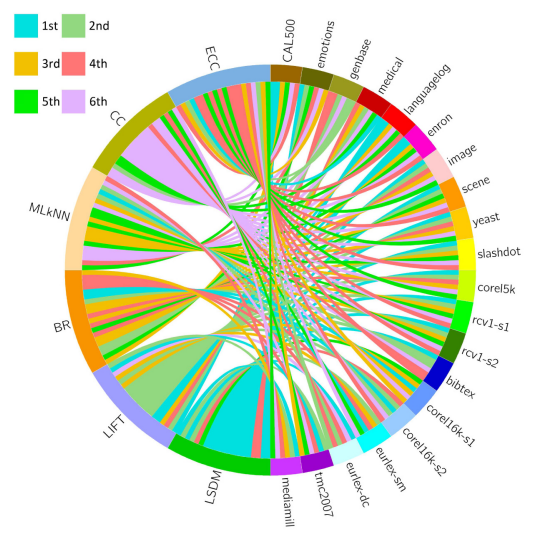

(c) Hamming loss

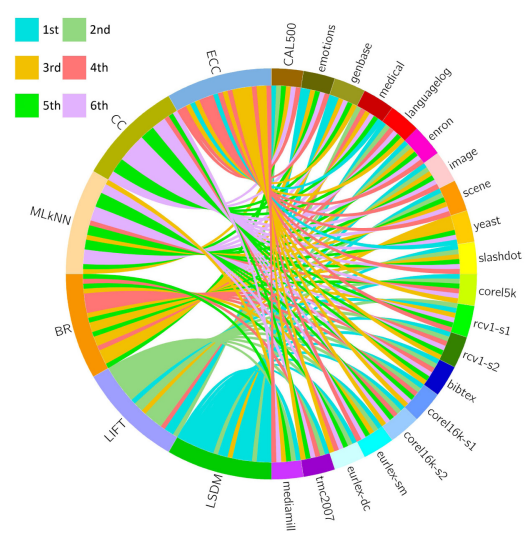

(e) One-error

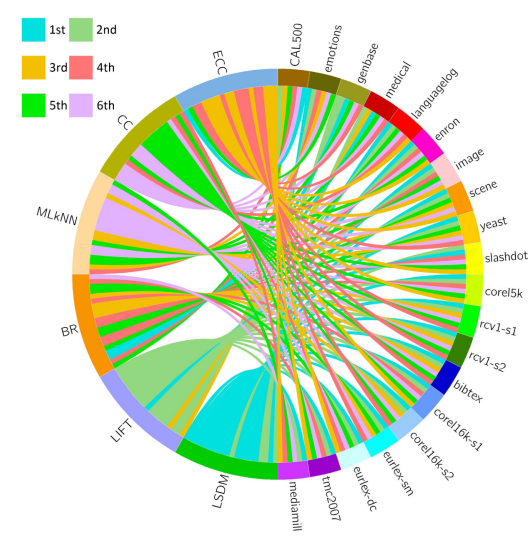

(b) Macro-averaging AUC

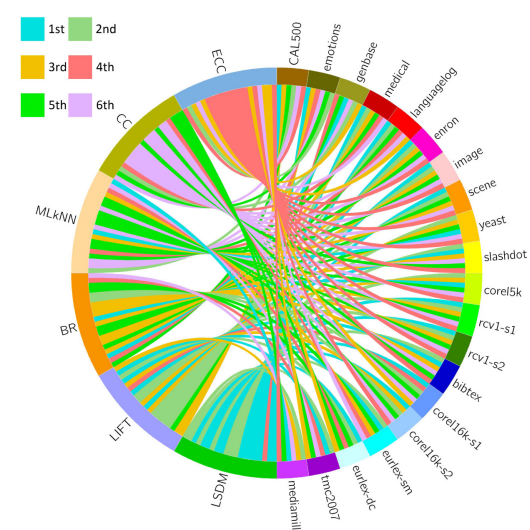

(d) Coverage

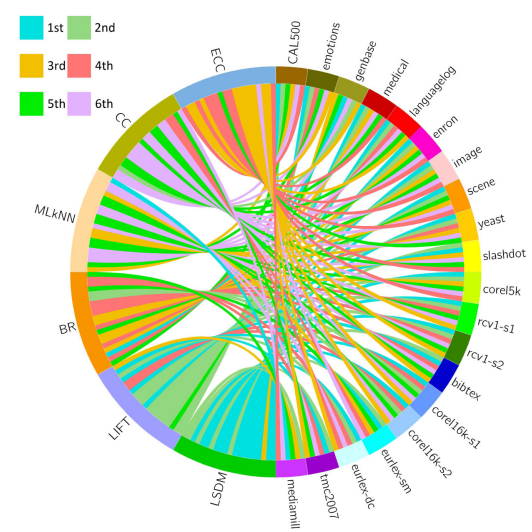

(f) Ranking loss

Fig. 4. Comparison of LSDM against other comparing algorithms under each evaluation measure. Each dataset connects all the algorithms with different color curves simultaneously and the size of identical color curve area on the left half circle denotes the rank performance of each algorithm corresponding to all datasets. 
other comparing algorithms, as shown in Figure 4 LSDM exceeding LIFT at most rank circumstances is superior. As LIFT can be viewed as a degenerated version of LSDM where the label-specific discriminant mapping features $\phi_{k}(\boldsymbol{x})$ is only kept to the distance mapping features $\phi_{k}^{\prime}(\boldsymbol{x})$. The superior performance of LSDM against LIFT clearly verifies the effectiveness of linear representation features and employing sLDA to excavate the optimally reconstructed feature space.

(2) As shown in Figures 3 and 4, LSDM achieves statistically superior or at least comparable performance against BR in terms of each evaluation measure. As BR can be regarded as a plain version of LSDM by keeping the original feature vector unchanged, the superior performance of LSDM against BR clearly verifies the effectiveness of employing labelspecific discriminant mapping features.

(3) Furthermore, LSDM significantly outperforms MLkNN, CC, and ECC in terms of each evaluation measure. Note that label transforming to feature learning strategy has been incorporated into $\mathrm{CC}$ and ECC to deal with the inherent randomness in their learning procedure, similar strategy may also be utilized by LSDM to account for the randomness in its procedure.

(4) As shown in Tables 3 and 4 and Figure 4, across all evaluation measures, LSDM ranks first in $71.2 \%$ cases on the datasets with sparse features (genbase, medical, language log, enron, slashdot, rcv1-s1, rcv1-s2, bibtex, eurlex-sm, eurlex-dc and tmc2007). On the other hand, LSDM ranks first in more than $85.2 \%$ cases on the datasets with dense features (CAL500, emotions, image, scene, yeast, corel5k, corel16k-s1, corel16k-s2 and mediamill). These results indicate that LSDM tends to work better in application domains with dense feature representation than those with sparse feature representation.

(5) Furthermore, across all evaluation measures, LSDM ranks first in $77.5 \%$ cases on all datasets with comparing algorithms. Especially, LSDM ranks first in $78.3 \%$ cases on regular-scale datasets (Table 3) and ranks first in $76.7 \%$ cases on large-scale datasets (Table 4). These results indicate that LSDM tends to work stably with arbitrary-scale datasets.

To summarize, LSDM achieves rather competitive performance against other well-established multi-label learning algorithms across 20 benchmark datasets and six evaluation measures, which validates the effectiveness of label-specific discriminant mapping features. Specifically, the linear representation features and sLDA to deal with the setting of diverse $\beta$ values show the superiority of LSDM compared with LIFT. The performance advantage is more pronounced on arbitrary-scale datasets with dense features.

\section{CONCLUSION}

In this article, we propose a novel algorithm named LSDM to deal with the multi-label learning problem. Previously, numerous multi-label algorithms learn from training examples by manipulating the label space, such as exploiting label correlations and reducing label space dimension. To deal with this problem, LIFT which is the pioneer to learn from training examples by manipulating the feature space, i.e., learning label specific features, conducts cluster analysis on the positive and negative instances with regard to each label and performs training and testing by querying the clustering results which are employed to construct distance mapping features. However, it has two drawbacks: (a) utilizing identical $\beta$ value to control the number of clusters for each label ignores the differences between labels; (b) using the distance information cannot exploit the clustering results comprehensively. To overcome these two drawbacks, LSDM sets diverse $\beta$ values to conduct cluster analysis for identical label and more thoroughly explores the label-specific information by using linear representation features which describe the spatial topological information. Due to the 
problem of diverse reconstructed feature spaces for identical label, it employs sLDA to excavate optimal one with regard to each label efficiently.

The major contribution of our work is to utilize label-specific discriminant mapping features, which suggests a promising direction for learning from multi-label data. Experiments across the largest number of benchmark datasets up to date show that (a) linear representation and sLDA effectively exploit label-specific information; (b) LSDM achieves highly competitive performance against other state-of-the-art multi-label learning algorithms; (c) multi-label learning algorithms comprising binary classifiers might be improved by utilizing label-specific discriminant mapping features.

In the future, it is interesting to design other strategies of generating label-specific discriminant mapping features which incorporate these features into other multi-label learning algorithms, and improve LSDM by considering label correlations into the feature construction step.

\section{REFERENCES}

[1] Zafer Barutçuoglu, Robert E. Schapire, and Olga G. Troyanskaya. 2006. Hierarchical multi-label prediction of gene function. Bioinformatics 22, 7 (2006), 830-836.

[2] Matthew R. Boutell, Jiebo Luo, Xipeng Shen, and Christopher M. Brown. 2004. Learning multi-label scene classification. Pattern Recognition 37, 9 (2004), 1757-1771.

[3] Ricardo Silveira Cabral, Fernando De la Torre, João Paulo Costeira, and Alexandre Bernardino. 2011. Matrix completion for multi-label image classification. In Proceedings of the Neural Information Processing Systems, Vol. 201. 2.

[4] Ricardo Silveira Cabral, Fernando De la Torre, João Paulo Costeira, and Alexandre Bernardino. 2015. Matrix completion for weakly-supervised multi-label image classification. IEEE Transactions on Pattern Analysis and Machine Intelligence 37, 1 (2015), 121-135.

[5] Nicolò Cesa-Bianchi, Matteo Re, and Giorgio Valentini. 2012. Synergy of multi-label hierarchical ensembles, data fusion, and cost-sensitive methods for gene functional inference. Machine Learning 88, 1-2 (2012), 209-241.

[6] Chih-Chung Chang and Chih-Jen Lin. 2011. LIBSVM: A library for support vector machines. ACM Transactions on Intelligent Systems and Technology 2, 3 (2011), 27.

[7] Li Li, Houfeng Wang, Xu Sun, Baobao Chang, Shi Zhao, and Lei Sha. 2015. Multi-label text categorization with joint learning predictions-as-features method. In Proceedings of the 2015 Conference on Empirical Methods in Natural Language Processing (EMNLP'15). 835-839.

[8] Weiwei Cheng and Eyke Hüllermeier. 2009. Combining instance-based learning and logistic regression for multilabel classification. Machine Learning 76, 2-3 (2009), 211-225.

[9] Amanda Clare and Ross D. King. 2001. Knowledge discovery in multi-label phenotype data. In Proceedings of the Principles of Data Mining and Knowledge Discovery. Springer, 42-53.

[10] Janez Demsar. 2006. Statistical comparisons of classifiers over multiple data sets. fournal of Machine Learning Research 7 (2006), 1-30.

[11] Olive Jean Dunn. 1961. Multiple comparisons among means. Journal of American Statistical Association 56, 293 (1961), $52-64$.

[12] Johannes Fürnkranz, Eyke Hüllermeier, Eneldo Loza Mencía, and Klaus Brinker. 2008. Multilabel classification via calibrated label ranking. Machine Learning 73, 2 (2008), 133-153.

[13] Yuhong Guo and Suicheng Gu. 2011. Multi-label classification using conditional dependency networks. In Proceedings of the 22nd International foint Conference on Artificial Intelligence. Barcelona, Catalonia, Spain. 1300-1305.

[14] James A. Hanley and Barbara J. McNeil. 1982. The meaning and use of the area under a receiver operating characteristic (ROC) curve. Radiology 143, 1 (1982), 29-36.

[15] Peng Hou, Xin Geng, and Min-Ling Zhang. 2016. Multi-label manifold learning. In Proceedings of the Thirtieth AAAI Conference on Artificial Intelligence. Phoenix, Arizona, USA. 1680-1686.

[16] Jun Huang, Guorong Li, Qingming Huang, and Xindong Wu. 2015. Learning label specific features for multi-label classification. In Proceedings of the IEEE International Conference on Data Mining. IEEE, 181-190.

[17] Jun Huang, Guorong Li, Qingming Huang, and Xindong Wu. 2016. Learning label-specific features and classdependent labels for multi-label classification. IEEE Transactions on Knowledge and Data Engineering 28, 12 (2016), 3309-3323.

[18] Jun Huang, Guorong Li, Qingming Huang, and Xindong Wu. 2018. Joint feature selection and classification for multilabel learning. IEEE Transactions on Cybernetics 48, 3 (2018), 876-889.

[19] Anil K. Jain, M. Narasimha Murty, and Patrick J. Flynn. 1999. Data clustering: A review. ACM Computing Surveys 31, 3 (1999), 264-323. 
[20] Shuiwang Ji, Lei Tang, Shipeng Yu, and Jieping Ye. 2010. A shared-subspace learning framework for multi-label classification. ACM Transactions on Knowledge Discovery from Data 4, 2 (2010), 8.

[21] Abhishek Kumar, Shankar Vembu, Aditya Krishna Menon, and Charles Elkan. 2012. Learning and inference in probabilistic classifier chains with beam search. In Proceedings of the Machine Learning and Knowledge Discovery in Databases. Springer, 665-680.

[22] Yu-Kun Li, Min-Ling Zhang, and Xin Geng. 2015. Leveraging implicit relative labeling-importance information for effective multi-label learning. In Proceedings of the IEEE International Conference on Data Mining. Atlantic City, N7, USA. 251-260.

[23] Eneldo Loza Mencía and Johannes Fürnkranz. 2008. Pairwise learning of multilabel classifications with perceptrons. In Proceedings of the International Joint Conference on Neural Networks, Part of the IEEE World Congress on Computational Intelligence. Hong Kong, China. 2899-2906.

[24] Gjorgji Madjarov, Dejan Gjorgjevikj, and Tomche Delev. 2011. Efficient two stage voting architecture for pairwise multi-label classification. In Proceedings of the Advances in Artificial Intelligence. Springer, 164-173.

[25] Elena Montañés, Robin Senge, José Barranquero, José Ramón Quevedo, Juan José del Coz, and Eyke Hüllermeier. 2014. Dependent binary relevance models for multi-label classification. Pattern Recognition 47, 3 (2014), 1494-1508.

[26] Lishan Qiao, Limei Zhang, Zhonggui Sun, and Xueyan Liu. 2017. Selecting label-dependent features for multi-label classification. Neurocomputing 259 (2017), 112-118.

[27] Jesse Read, Bernhard Pfahringer, and Geoff Holmes. 2008. Multi-label classification using ensembles of pruned sets. In Proceedings of the Eighth IEEE International Conference on Data Mining. IEEE, 995-1000.

[28] Jesse Read, Bernhard Pfahringer, Geoff Holmes, and Eibe Frank. 2011. Classifier chains for multi-label classification. Machine Learning 85, 3 (2011), 333-359.

[29] Sam T. Roweis and Lawrence K. Saul. 2000. Nonlinear dimensionality reduction by locally linear embedding. Science 290, 5500 (2000), 2323-2326.

[30] Timothy N. Rubin, America Chambers, Padhraic Smyth, and Mark Steyvers. 2012. Statistical topic models for multilabel document classification. Machine Learning 88, 1-2 (2012), 157-208.

[31] Robert E. Schapire and Yoram Singer. 2000. BoosTexter: A boosting-based system for text categorization. Machine Learning 39, 2/3 (2000), 135-168.

[32] Lu Sun, Mineichi Kudo, and Keigo Kimura. 2016. Multi-label classification with meta-label-specific features. In Proceedings of the 23rd International Conference on Pattern Recognition. IEEE, 1612-1617.

[33] Grigorios Tsoumakas, Ioannis Katakis, and Ioannis Vlahavas. 2010. Mining multi-label data. In Proceedings of the Data Mining and Knowledge Discovery Handbook. Springer, 667-685.

[34] Grigorios Tsoumakas, Ioannis Katakis, and Ioannis P. Vlahavas. 2011. Random k-labelsets for multilabel classification. IEEE Transactions on Knowledge and Data Engineering 23, 7 (2011), 1079-1089.

[35] G. Tsoumakas, M. L. Zhang, and Z. H. Zhou. 2009. Tutorial on learning from multi-label data. In Proceedings of the European Conference on Machine Learning and Principles and Practice of Knowledge Discovery in Databases.

[36] Alexis Vallet and Hiroyasu Sakamoto. 2015. A multi-label convolutional neural network for automatic image annotation. Journal of Information Processing 23, 6 (2015), 767-775.

[37] Xiao Wang, Jun Zhang, and Guo-Zheng Li. 2015. Multi-location gram-positive and gram-negative bacterial protein subcellular localization using gene ontology and multi-label classifier ensemble. BMC Bioinformatics 16, Suppl 12 (2015), S1.

[38] Wei Weng, Yaojin Lin, Shunxiang Wu, Yuwen Li, and Yun Kang. 2018. Multi-label learning based on label-specific features and local pairwise label correlation. Neurocomputing 273 (2018), 385-394.

[39] Suping Xu, Xibei Yang, Hualong Yu, Dong-Jun Yu, Jingyu Yang, and Eric C. C. Tsang. 2016. Multi-label learning with label-specific feature reduction. Knowledge-Based Systems 104 (2016), 52-61.

[40] Ju-Jie Zhang, Min Fang, and Xiao Li. 2015. Multi-label learning with discriminative features for each label. Neurocomputing 154 (2015), 305-316.

[41] Min-Ling Zhang. 2011. LIFT: Multi-label learning with label-specific features. In Proceedings of the 22nd International foint Conference on Artificial Intelligence. Barcelona, Catalonia, Spain. 1609-1614.

[42] Min-Ling Zhang and Lei Wu. 2015. Lift: Multi-label learning with label-specific features. IEEE Transactions on Pattern Analysis and Machine Intelligence 37, 1 (2015), 107-120.

[43] Min-Ling Zhang and Kun Zhang. 2010. Multi-label learning by exploiting label dependency. In Proceedings of the 16th ACM SIGKDD International Conference on Knowledge Discovery and Data Mining. Washington, DC, USA, 999-1008.

[44] Min-Ling Zhang and Zhi-Hua Zhou. 2007. ML-KNN: A lazy learning approach to multi-label learning. Pattern Recognition 40, 7 (2007), 2038-2048.

[45] Min-Ling Zhang and Zhi-Hua Zhou. 2014. A review on multi-label learning algorithms. IEEE Transactions on Knowledge and Data Engineering 26, 8 (2014), 1819-1837.

Received March 2018; revised February 2019; accepted February 2019 